\title{
Removal of totally implanted venous access ports for suspected infection in the intensive care unit: a multicenter observational study
}

\author{
Marie Lecronier ${ }^{1 *}\left(\mathbb{D}\right.$, Sandrine Valade ${ }^{2}$, Naike Bigé ${ }^{3}$, Nicolas de Prost ${ }^{4}$, Damien Roux $^{5}$, \\ David Lebeaux ${ }^{6,7}$, Eric Maury ${ }^{3}$, Elie Azoulay ${ }^{2}$, Alexandre Demoule ${ }^{1,8}$, Martin Dres ${ }^{1,8}$ \\ and on behalf of the GrrrOH (Group for Research in Respiratory Intensive Care Onco-Hematology)
}

\begin{abstract}
Background: While no data support this practice, international guidelines recommend the removal of totally implanted venous access ports (TIVAPS) in patients with suspicion of TIVAP-related bloodstream infection admitted in the intensive care unit (ICU) for a life-threatening sepsis.

Methods: During this multicenter, retrospective and observational study, we included all patients admitted in five ICU for a life-threatening sepsis in whom a TIVAP was removed between January 2012 and December 2014. We aimed (1) at determining the proportion of confirmed TIVAP-related infections and (2) at assessing short- and long-term survival of patients with and without TIVAP-related infections.

Results: One hundred and fifty-one patients (58 \pm 14 years, 62\% males) were included between 2012 and 2014. TIVAP-related infections were confirmed in 68 patients (45\%). Demographic characteristics were similar between patients with and without TIVAP-related infections. SOFA score on admission per point increase [odd ratio (OR), 0.86 interval confidence (IC) 95\% (0.8-0.9), $p<0.01$ ] and local signs of infection [OR 4.0, IC 95\% (1.1-15.6), $p=0.04$ ] were significantly associated with TIVAP-related infection. Patients with TIVAP-related infection had lower ICU and 6-month mortality as compared to their counterparts ( 9 vs. $40 \%$, respectively, $p<0.01$; and $50 \mathrm{vs.} 66 \%$, respectively, $p=0.04$ ). TIVAP-related infection was significantly associated with ICU survival [OR 0.2, IC 95\% $(0.05-0.5), p<0.01]$.
\end{abstract}

Conclusions: TIVAP-related infection was confirmed in nearly one out of two cases of life-threatening sepsis in patients in whom it has been removed. TIVAP-related infection was associated with a good prognosis, as compared to patients with other causes of infection.

Keywords: Sepsis, Intensive care unit, Totally implantable venous access ports

\section{Background}

Totally implanted venous access ports (TIVAPs) are commonly used for patients requiring long-term or iterative treatments such as antineoplastic chemotherapy,

\footnotetext{
*Correspondence: marie.lecronier@aphp.fr

1 Service de Pneumologie et Réanimation Médicale (Département "R3S"), Groupe Hospitalier Pitié-Salpêtrière Charles Foix, Assistance PubliqueHôpitaux de Paris, 75013 Paris, France

Full list of author information is available at the end of the article
}

parenteral nutrition and transfusion [1-3]. Even if TIVAPs are associated with a low risk of infection, they still remain a source of infections potentially leading to life-threatening sepsis and subsequent admission in the intensive care unit (ICU) $[4,5]$.

In case of tunnel or port-pocket infection, TIVAPrelated bloodstream infection is obviously strongly suspected and the device should be promptly removed [6]. However, local signs of infection are frequently lacking [7-10]. International guidelines support the removal 
of TIVAP in case of TIVAP-related bloodstream infection, with complication like severe sepsis or/and septic shock (use of vasopressors) [6,11], although no data support this practice. Removal of TIVAP may have deleterious consequences in critically ill patients. First, patients with TIVAP are frequently frail and exposed to uncontrolled bleeding (low platelets and coagulation disorders). Second, removal of TIVAP is a surgical procedure that may interfere with the management of the ongoing sepsis [12-15]. Eventually, removing TIVAP may defer administration of chemotherapy or specific treatments once patients are discharged from the ICU. Therefore, removal of TIVAP is an important decision that should be supported by clinical evidences, but predictive factors of TIVAP-related infections are lacking in ICU patients. Likewise, no study regarding the prognosis of TIVAPrelated infections has been conducted outside the ICU [16-20]. In light with this, the present study was designed to address three main objectives: (1) to determine the proportion of confirmed TIVAP-related infections in a population of patients admitted in the ICU in whom a TIVAP was removed for life-threatening sepsis, (2) to identify predictive factors of confirmed TIVAP infection in patients admitted to the ICU and (3) to assess shortand long-term outcome of patients with TIVAP-related infection and compare them with their counterparts in whom TIVAP was removed without confirmation of infection.

\section{Patients and methods}

This retrospective, multicenter, observational study was conducted in five ICU located in academic hospitals. The study period extended from January 2012 through December 2014. The Institutional Review Board of the French Intensive Care Society approved the study (CE SRLF15-52).

\section{Selection of patients}

Using clinical microbiology laboratory databases, we identified retrospectively all the patients admitted in participating ICU in whom a TIVAP was removed during the ICU stay and sent to the microbiology laboratory. Each patient's record was analyzed by two investigators (ML and MD) and those patients who fulfilled the following criteria were entered into the study: (1) age $>18$ years, (2) sepsis, severe sepsis or septic shock, defined according to criteria of the Surviving Sepsis Campaign's definition [21], as the main reason for TIVAP removal. Of notice, peripherally inserted central catheters and surgically inserted long-term central venous catheters others than TIVAP were not considered for the study. In addition, patients with TIVAP removed before ICU admission or for another reason than sepsis (thrombosis, uselessness) were also not included in the study.

\section{Data collection}

The following data were extracted from each patient's medical record: age, gender, clinical and biological variables on admission. Simplified Acute Physiology Score (SAPS) 2 [22] and Sequential Organ Failure Assessment (SOFA) [23] were calculated upon ICU admission. Predisposing risk factors for TIVAP-related infections were also collected: immunosuppression status (i.e., hematological malignancies, solid organ transplant, recent antineoplastic chemotherapy for cancer or HIV infection), time since TIVAP insertion, main indication of the TIVAP (antineoplastic chemotherapy, parenteral nutrition), date of last antineoplastic chemotherapy and delay between ICU admission and removal of the device. We also looked through each patient's record for local signs of infection (induration or erythema, warmth and pain or tenderness along the tract of a catheterized vein) whenever it was described and general sign of infection (fever defined as temperature $\geq 38^{\circ} 3$, hypothermia defined as temperature $<36^{\circ}$, hypotension defined as systolic blood pressure $<90 \mathrm{mmHg}$ or mean blood pressure $<65 \mathrm{mmHg}$ ). Advanced life support measures taken during the ICU stay (mechanical ventilation and vasopressors) and antibiotics regimens were recorded for each patient. Microbiological data were collected as follows: positive culture of TIVAP catheter tip or port reservoir, positive blood culture from the TIVAP and from a peripheral vein with the differential time to positivity. We also collected information regarding use of appropriate antibiotic in initial regimen (antibiotics with in vitro activity against the infecting agent). Finally, we recorded length of ICU stay, time spent under invasive mechanical ventilation and vasopressors. Mortality was determined in the ICU, at 28 days and 6 months after ICU admission.

\section{Definitions}

Culture of TIVAP was considered as positive if the tip or port reservoir (indoor or outdoor) was positive. Positivity of TIVAP tip culture was defined according to the same modality across all the microbiological laboratories of participating centers. It was defined on blood agar plate by quantitative method after vortexing or sonication (taking into account pathogens present in their inner or outer surfaces) with a cutoff of $\geq 1000$ colony-forming units $(\mathrm{CFU}) / \mathrm{mL}$ [24-26]. Growth of $<1000 \mathrm{CFU} / \mathrm{mL}$ from a catheter by quantitative method was considered as catheter contamination. TIVAP box culture was performed according to each clinical microbiology laboratory-own protocol, such as immersion of the case in broth and then sowing on blood agar plate or chocolate plate, needle 
puncture and aspiration of the case contents then sowing, or swab from outside the case then sowing. In all these techniques, a qualitative culture was performed.

Definition of TIVAP-related infection was adapted from the Infectious Diseases Society of America (IDSA) guidelines [6] as one of the following conditions:

1. TIVAP-related bloodstream infection, defined as (1) a positive culture of the TIVAP (catheter tip or reservoir's port) associated with a positive peripheral blood culture with the same microorganism (same species and same antibiotic susceptibility testing) or (2) a differential time to positivity of a blood culture drawn from the catheter versus from a peripheral vein (positivity of the catheter blood sample at least $2 \mathrm{~h}$ before the peripheral blood sample) [27, 28];

2. Local or general (fever $\geq 38^{\circ} 3$ or $<36^{\circ}$ and chills) signs of infection, positive culture of TIVAP (catheter tip or the reservoir's port) and regression of clinical signs of infection after TIVAP removal despite a negative peripheral blood culture.

We also included patients who did not meet the two above conditions but who had positive blood culture without other suspected infection and regression of clinical signs of infection after TIVAP removal despite negative culture of TIVAP (catheter tip or the reservoir's port).

Exclusive TIVAP-related infection was defined by TIVAP-related infection that was not associated with any other documented source of infection among lower respiratory, digestive or urinary tract infection.

\section{Statistical analysis}

Continuous variables are expressed as mean \pm standard deviation or median (interquartile range). Categorical variables are expressed as number and relative frequencies. Patients were categorized a posteriori into two groups according to microbiologic findings: patients with or without TIVAP-related infection. Continuous variables were tested for normality using the Shapiro-Wilk test. Gaussian variables were compared using a $t$ test and non-normally distributed variables using a Mann-Whitney test. Categorical variables were compared with Chisquare test. The primary endpoint was the prevalence of TIVAP-related infection. A stepwise logistic regression analysis was performed to identify variables associated with TIVAP-related infection and with ICU and 28-day mortality. Variables found to have univariate association $(p<0.05)$ with the outcome of interest were considered in the final model. Kaplan-Meier survival curves for patients with and without TIVAP-related infections were computed for 6-month mortality. For all final comparisons, a two-tailed $p$ value less than or equal to 0.05 was considered statistically significant. The statistical analysis was performed with SAS statistical V9.3 software (SAS Institute Inc., Cary, NC, USA).

\section{Results \\ Characteristics upon ICU admission}

Over the study period, 151 patients met the inclusion criteria and were retained in the analysis (see flowchart, Additional file 1: Figure S1). Main characteristics of the patients are presented in Table 1. One hundred and fortyeight (98\%) patients were immunosuppressed, and 50 patients (33\%) had neutropenia. Antineoplastic chemotherapy was administrated in 131 patients $(87 \%)$ in the last 6 months, and $18(12 \%)$ of the patients were receiving parenteral nutrition. Severe sepsis was present in 48 patients (32\%) and septic shock in 93 (62\%).

\section{Proportion and features of TIVAP-related infection}

TIVAP-related infection was found in 68 patients (45\%). Among these 68 patients, the diagnosis of TIVAP-related infection was retained because of (1) TIVAP-related bloodstream infection with the association of a positive peripheral blood culture with a positive culture of the TIVAP (tip or reservoir) in 33 patients (48\%) or a differential time to positivity of a blood culture drawn from the catheter versus from a peripheral vein in 11 patients (16\%); (2) a positive culture of the TIVAP and the regression of clinical signs of infection after TIVAP removal in $12(18 \%)$ patients (negative peripheral blood culture) or (3) a positive blood culture associated with favorable outcome after TIVAP removal in $12(18 \%)$ patients (negative tip or reservoir culture of the TIVAP). A growth of $100 \mathrm{CFU} / \mathrm{mL}$ of Staphylococcus epidermidis was found in two patients who were classified as catheter contamination (without TIVAP-related infection).

Regarding microbiological findings, TIVAP-related infections were associated with 53\% (36/68) of Gramnegative rods, $44 \%$ (30/68) of Gram-positive cocci and 7\% (5/68) of Candida sp. (see Table 2). Sixty-nine percent of patients with TIVAP-related infection had no other focus of infection and were subsequently classified as exclusive TIVAP-related infection (see Additional file 1: Table S1). Among patients with infectious other than TIVAPrelated infections, $65 \%$ had positive microbiological samples (see Table 3; Additional file 1: Table S2).

\section{Characteristics of the patients according to the presence or absence of TIVAP-related infections}

Demographic characteristics of patients with and without TIVAP-related infections were not different (Table 1). Parenteral nutrition was more frequent in patients with TIVAP-related infections. In addition, SAPS2 and SOFA 
Table 1 Clinical characteristics and laboratory features upon intensive care unit admission

\begin{tabular}{|c|c|c|c|c|}
\hline Characteristic & $\begin{array}{l}\text { All } \\
n=151\end{array}$ & $\begin{array}{l}\text { TIVAP-related infection } \\
n=68\end{array}$ & $\begin{array}{l}\text { No TIVAP-related infection } \\
n=83\end{array}$ & $p$ value \\
\hline Age, year & $58 \pm 14$ & $57 \pm 14$ & $58 \pm 14$ & 0.58 \\
\hline Female gender, $n(\%)$ & $58(38)$ & $30(44)$ & $28(34)$ & 0.19 \\
\hline Transfer from the emergency room, $n(\%)$ & $47(31)$ & $21(31)$ & $26(31)$ & 1.00 \\
\hline Transfer from the ward, $n(\%)$ & $104(69)$ & $47(69)$ & $57(69)$ & 1.00 \\
\hline SAPS2 & $52 \pm 17$ & $47 \pm 15$ & $56 \pm 17$ & $<0.01$ \\
\hline SOFA & $9 \pm 4$ & $7 \pm 4$ & $10 \pm 4$ & $<0.01$ \\
\hline \multicolumn{5}{|l|}{ TIVAP-related infection risk factors, $n$ (\%) } \\
\hline Immunosuppression & $148(98)$ & $66(97)$ & $82(99)$ & 0.44 \\
\hline Hematological malignancies & $72(48)$ & $28(41)$ & $44(53)$ & 0.14 \\
\hline Solid organ cancer & $71(47)$ & $35(51)$ & $36(43)$ & 0.32 \\
\hline Metastatic cancer & $44(29)$ & $24(35)$ & $20(24)$ & 0.13 \\
\hline Recent chemotherapy (< 6 months) & $131(87)$ & $58(85)$ & $73(88)$ & 0.63 \\
\hline Parenteral nutrition & $18(12)$ & $14(21)$ & $4(5)$ & $<0.01$ \\
\hline \multicolumn{5}{|l|}{ Clinical signs } \\
\hline Temperature $<36$ or $\geq 38.3^{\circ} \mathrm{C}, n(\%)$ & $111(74)$ & $55(81)$ & $56(67)$ & 0.08 \\
\hline Systolic blood pressure, $\mathrm{mmHg}$ & $98 \pm 27$ & $97 \pm 29$ & $99 \pm 25$ & 0.54 \\
\hline Mean blood pressure, mmHg & $69 \pm 20$ & $69 \pm 23$ & $70 \pm 18$ & 0.77 \\
\hline Glasgow Score Scale & $13 \pm 3$ & $14 \pm 3$ & $13 \pm 4$ & 0.04 \\
\hline Local sign of infection, $n$ (\%) & $15(10)$ & $12(18)$ & $3(4)$ & $<0.01$ \\
\hline \multicolumn{5}{|l|}{ Biological signs } \\
\hline White blood cells < 1 Giga/l, $n$ (\%) & $50(33)$ & $19(28)$ & $31(37)$ & 0.22 \\
\hline Platelet counts, Giga/l & $116 \pm 113$ & $124 \pm 99$ & $110 \pm 123$ & 0.45 \\
\hline Prothrombin time, $\%$ & $64 \pm 17$ & $69 \pm 17$ & $60 \pm 17$ & $<0.01$ \\
\hline Serum creatinine, $\mu \mathrm{mol} / / \mathrm{I}$ & $142 \pm 119$ & $126 \pm 113$ & $155 \pm 123$ & 0.13 \\
\hline Bicarbonate, $\mathrm{mmol} / \mathrm{l}$ & $20 \pm 5$ & $21 \pm 5$ & $20 \pm 6$ & 0.05 \\
\hline Arterial lactate, mmol// & $3.4 \pm 3.2$ & $3.3 \pm 3.0$ & $3.6 \pm 3.3$ & 0.61 \\
\hline
\end{tabular}

Categorical variables are expressed as no. (\%) and continuous variables as mean $\pm S D$

SAPS2 Simplified Acute Physiology Score, SOFA Sepsis-Related Organ Failure Assessment, TIVAP totally implanted venous access port

score were higher in patients with infectious other than TIVAP-related infections as compared to their counterparts. Local signs of infection were present in $18 \%$ of the patients with a TIVAP-related infection versus $4 \%$ for their counterparts $(p<0.01)$. There was no difference regarding the presence of neutropenia upon admission between patients with TIVAP-related infection and patients without.

By multivariate logistic regression analysis, two factors were independently associated with TIVAP-related infection: SOFA score upon admission per point increase [odd ratio (OR) 0.86 interval confidence (IC) 95\% (0.80-0.90), $p<0.01$ ] and local signs of infection [OR 4.0 IC 95\% (1.115.6), $p=0.04]$.

\section{Therapeutic management and outcome}

Patients with infections other than TIVAP-related infections received more vasopressors and were more likely to require mechanical ventilation than their counterparts (Table 4). There was no significant difference in terms of antibiotics management between both groups. The duration of ICU stay was similar between patients with and without TIVAP-related infections. Overall ICU mortality was $26 \%$. Patients with TIVAP-related infection had lower ICU, 28-day and 6-month mortality as compared to their counterparts (Table 4; Fig. 1). In addition, patients with exclusive TIVAP-related infection had a lower ICU mortality as compared to patients who had TIVAP-related infection and another focus of infection: $2 \%(1 / 47)$ versus $24 \%(5 / 21)$, respectively $(p<0.01)$.

Table 5 displays the variables associated with 28 -day mortality, and Tables 2 and 3 show microbiological findings associated with 28-day mortality among patients with and without TIVAP-related infections, respectively. By multivariate logistic regression analysis, three factors were independently associated with higher 28-day mortality: SOFA at admission per point increase [OR 1.3 IC 95\% (1.1-1.6), $p<0.01$ ], the use of mechanical ventilation [OR 11 IC 95\% (2.8-41.2), $p<0.01$ ] and hematological malignancies [OR 3.2 IC 95\% (1.1-9.1), $p=0.03$ ]. 
Table 2 Microbiological findings in patients with totally implanted venous access ports-related infections and association with 28-day mortality (univariate analysis)

\begin{tabular}{lccll}
\hline & $\begin{array}{l}\text { All } \\
\boldsymbol{n = 6 8}\end{array}$ & $\begin{array}{l}\text { Alive } \\
\boldsymbol{n}=\mathbf{5 4}\end{array}$ & $\begin{array}{l}\text { Dead } \\
\boldsymbol{n}=\mathbf{1 4}\end{array}$ & $\boldsymbol{p}$ value \\
\hline Gram-negative bacilli, $n(\%)$ & $36(53)$ & $32(59)$ & $4(29)$ & 0.07 \\
Enterobacteriaceae & $27(40)$ & $25(46)$ & $2(14)$ & 0.03 \\
Escherichia coli & $8(12)$ & $8(15)$ & $0(0)$ & 0.19 \\
Klebsiella pneumoniae & $7(10)$ & $6(11)$ & $1(7)$ & 0.99 \\
Enterobacter cloacae & $7(10)$ & $7(13)$ & $0(0)$ & 0.33 \\
Other Enterobacteriaceae & $6(9)$ & $5(9)$ & $1(7)$ & 0.99 \\
Pseudomonas aeruginosa & $8(12)$ & $6(11)$ & $2(14)$ & 0.99 \\
Stenotrophomonas maltophilia & $1(2)$ & $1(2)$ & $0(0)$ & 0.99 \\
Acinetobacter sp. & $1(2)$ & $1(2)$ & $0(0)$ & 0.99 \\
Gram-positive cocci, $n$ (\%) & $30(44)$ & $21(39)$ & $9(64)$ & 0.13 \\
Staphylococcus aureus & $9(13)$ & $5(9)$ & $4(29)$ & 0.09 \\
Coagulase-negative staphylococci & $19(28)$ & $15(28)$ & $4(29)$ & 0.99 \\
Enterococcus sp. & $2(3)$ & $1(2)$ & $1(7)$ & 0.37 \\
Other Gram-positive cocci & $1(2)$ & $1(2)$ & $0(0)$ & 0.99 \\
Candida sp., $n$ (\%) & $5(7)$ & $2(4)$ & $3(21)$ & 0.06 \\
Polymicrobial, $n$ (\%) & $6(9)$ & $4(7)$ & $2(14)$ & 0.59 \\
\hline
\end{tabular}

Data are expressed as $n(\%)$

Table 3 Microbiological findings in patients without TIVAP (totally implanted venous access port)-related infections and association with 28-day mortality (univariate analysis)

\begin{tabular}{lccll}
\hline & $\begin{array}{l}\text { All } \\
\boldsymbol{n}=\mathbf{8 3}\end{array}$ & $\begin{array}{l}\text { Alive } \\
\boldsymbol{n}=\mathbf{4 3}\end{array}$ & $\begin{array}{l}\text { Dead } \\
\boldsymbol{n}=\mathbf{4 0}\end{array}$ & $\boldsymbol{p}$ value \\
\hline Gram-negative bacilli, $n$ (\%) & $34(41)$ & $17(40)$ & $17(43)$ & 0.83 \\
Enterobacteriaceae & $26(31)$ & $15(35)$ & $11(28)$ & 0.49 \\
Escherichia coli & $14(17)$ & $7(16)$ & $7(18)$ & 0.99 \\
Klebsiella pneumoniae & $7(9)$ & $4(9)$ & $3(8)$ & 0.99 \\
Enterobacter cloacae & $3(4)$ & $2(5)$ & $1(3)$ & 0.99 \\
Other Enterobacteriaceae & $9(11)$ & $7(16)$ & $2(5)$ & 0.16 \\
Pseudomonas aeruginosa & $6(7)$ & $1(2)$ & $5(13)$ & 0.10 \\
Acinetobacter sp. & $2(3)$ & $1(2)$ & $1(3)$ & 0.99 \\
Gram-positive cocci, $n$ (\%) & $22(27)$ & $8(19)$ & $14(35)$ & 0.13 \\
Staphylococcus aureus & $4(5)$ & $2(5)$ & $2(5)$ & 0.99 \\
Coagulase-negative staphylococci & $4(5)$ & $0(0)$ & $4(10)$ & 0.05 \\
Enterococcus sp. & $10(12)$ & $3(7)$ & $7(18)$ & 0.18 \\
Other Gram-positive cocci & $5(6)$ & $3(7)$ & $2(5)$ & 0.99 \\
Other bacteria, $n$ (\%) & $4(5)$ & $4(9)$ & $0(0)$ & 0.12 \\
Candida sp., $n$ (\%) & $8(10)$ & $4(9)$ & $4(10)$ & 0.99 \\
Polymicrobial, $n$ (\%) & $16(19)$ & $7(16)$ & $9(23)$ & 0.58 \\
\hline
\end{tabular}

Data are expressed as $n(\%)$

One factor, TIVAP-related infection, was independently associated with lower 28-day mortality [OR 0.2 IC 95\% (0.1-0.7), $p=0.02]$.
Additional file 1: Table S3 displays the variables associated with ICU mortality. By multivariate logistic regression analysis, two factors were independently associated with higher ICU mortality: SOFA at admission per point increase [OR 1.4 IC 95\% (1.2-1.7), $p<0.01$ ] and the use of mechanical ventilation [OR 14.0 IC 95\% (3.6-56.0), $p<0.01]$. One factor, TIVAP-related infection, was independently associated with lower ICU mortality [OR 0.16 IC 95\% (0.05-0.5), $p<0.01$.

\section{Discussion}

This study is seemingly the first reporting characteristics and outcome of patients admitted for a life-threatening sepsis in the ICU whose TIVAP has been removed. Our findings can be summarized as follows: (1) TIVAPrelated infection was confirmed in almost half of the patients; (2) except for a low value of SOFA score and local signs of infection, no other variable was found to be independently associated with TIVAP-related infection upon admission; and (3) patients with TIVAP-related infection had a better prognosis than patients with other source of infection.

\section{Proportion of TIVAP-related infection}

The proportion of patients in whom a TIVAP-related infection was confirmed was $45 \%$. Being the first study addressing this issue in the ICU, our results are hardly comparable. Indeed, most of previous studies were conducted outside the ICU $[4,5,10,17,19,20,29]$. For instance, in two studies $[8,9]$, among patient whose TIVAP was removed because of a suspected infection, TIVAP-related infection was confirmed in two thirds of the patients (19/29 and $15 / 23$, respectively). Based on our findings, the remaining key question is whether this $45 \%$ rate is high enough to justify TIVAP removal as supported by international guidelines [6] or whether these recommendations should be questioned. A higher rate $(>80 \%)$ would have made clearly acceptable the TIVAP removal (and all its consequences), whereas a lower rate $(<20 \%)$ would have discouraged current practices in term of potential harmful effects. While this $45 \%$ rate of TIVAP-related infection could be considered disappointing, it might represent the lower bounder of the proportion of patients with TIVAP-related infection. Indeed, all our patients received antibiotics prior ICU admission, a condition that could easily lead to false-negative microbiological findings [30]. As a matter of fact, this could have artificially underestimated the rate of patients with confirmed TIVAP-related infections. A randomized controlled trial might be necessary to definitively support the removal of TIVAP in case of severe sepsis or septic shock.

Another important finding is that a concomitant infection focus was evidenced in approximately one-third of 
Table 4 Therapeutic management and outcome of patients with and without TIVAP (totally implanted venous access port)-related infection

\begin{tabular}{|c|c|c|c|c|}
\hline Characteristic & $\begin{array}{l}\text { All patients } \\
n=151\end{array}$ & $\begin{array}{l}\text { TIVAP-related infections } \\
n=68\end{array}$ & $\begin{array}{l}\text { No TIVAP-related infections } \\
n=83\end{array}$ & $p$ value \\
\hline Time between ICU admission and TIVAP withdrawal, days & $1(0-2)$ & $1(0-1)$ & $1(0-2)$ & 0.09 \\
\hline \multicolumn{5}{|l|}{ Antibiotics } \\
\hline Beta lactam antibiotic, $n(\%)$ & $143(95)$ & $63(93)$ & $80(96)$ & 0.49 \\
\hline Glycopeptide/linezolid, n (\%) & $78(52)$ & $38(57)$ & $40(48)$ & 0.29 \\
\hline Use of $M V, n(\%)$ & $74(49)$ & $24(35)$ & $50(60)$ & $<0.01$ \\
\hline MV duration, days & $0(0-5)$ & $0(0-3)$ & $2(0-7)$ & 0.26 \\
\hline Vasopressors, $n(\%)$ & $103(68)$ & $37(55)$ & $66(80)$ & $<0.01$ \\
\hline Vasopressors, days & $2(0-3)$ & $1(0-2)$ & $2(1-4)$ & 0.04 \\
\hline ICU length of stay, days & $5(3-10)$ & $4(3-6)$ & $6(3-12)$ & 0.40 \\
\hline ICU mortality, n (\%) & $39(26)$ & $6(9)$ & $33(40)$ & $<0.01$ \\
\hline 28-day mortality, n (\%) & $54(36)$ & $14(20.5)$ & $40(48)$ & $<0.01$ \\
\hline 6-Month mortality, n (\%) & $89(59)$ & $34(50)$ & $55(66)$ & 0.04 \\
\hline
\end{tabular}

Categorical variables are expressed as no. (\%) and continuous variables as median (interquartile range)

TIVAP totally implanted venous access port, ICU intensive care unit, $M V$ mechanical ventilation

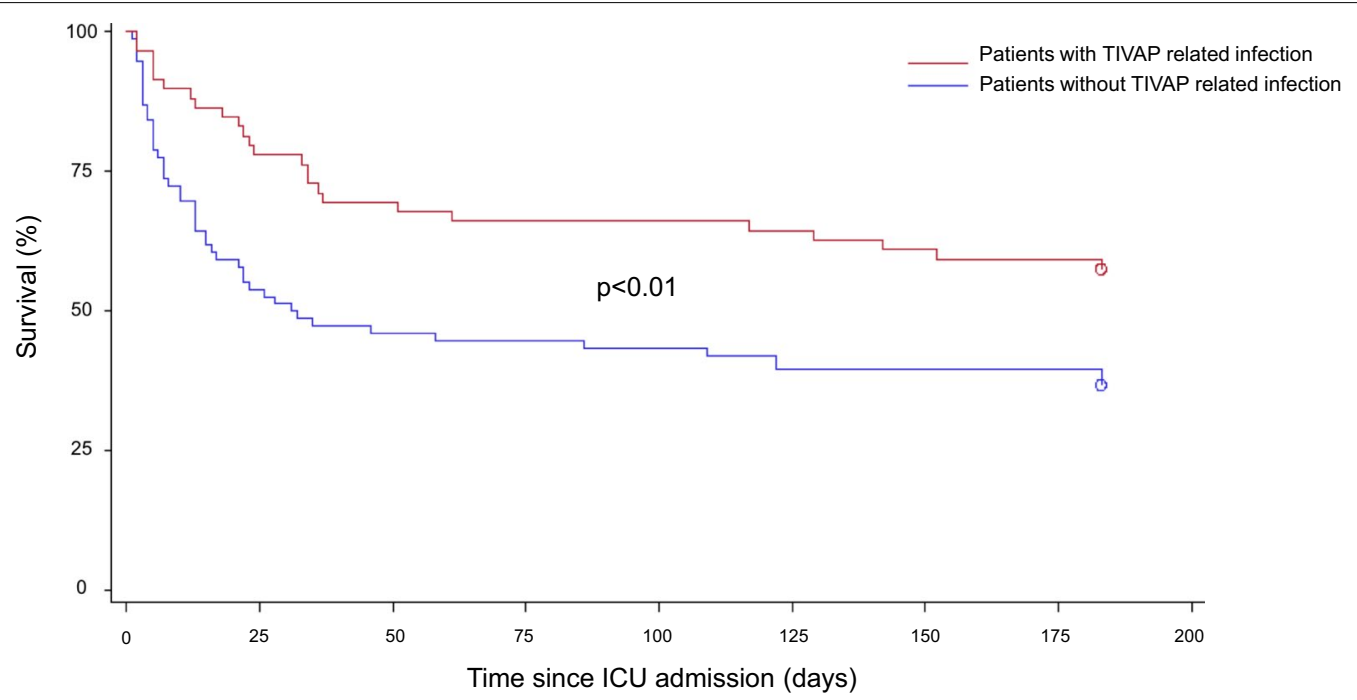

Fig. 1 Kaplan-Meier survival curve with 6-month mortality in patients with and without totally implanted venous access port (TIVAP)-related infections

patients with TIVAP-related infection. In half of these cases, the same pathogen was responsible (Additional file 1: Table S1). Whether this focus was the primary source or an hematogenous seeding from the TIVAPrelated infection is unknown. This finding points out the importance of an extensive investigation even though a TIVAP infection is identified.

\section{Factors associated with TIVAP-related infection}

While a $45 \%$ rate of confirmed TIVAP-related infection may be acceptable, identifying associated factors of
TIVAP-related infection upon admission would be helpful to prevent patients from undue procedures. Unfortunately, our study failed at providing new insightful clues in this purpose. Immunosuppression, a known risk factor for infection [4, 31-33], was equally present in both groups. By contrast, the use of parenteral nutrition, another recognized risk factor for TIVAP-related infection [34-36], was used more frequently in patients with TIVAP-related infection, but this finding remained infrequent and was not confirmed in multivariate analysis. While local signs of infection have been reported to be 
Table 5 Variables associated with 28-day mortality

\begin{tabular}{|c|c|c|c|c|}
\hline & $\begin{array}{l}\text { All } \\
n=151\end{array}$ & $\begin{array}{l}\text { Alive } \\
n=97\end{array}$ & $\begin{array}{l}\text { Dead } \\
n=54\end{array}$ & $p$ value \\
\hline Age, year & $58 \pm 14$ & $57 \pm 15$ & $59 \pm 13$ & 0.40 \\
\hline Female gender, $n(\%)$ & $58(38)$ & $41(43)$ & $17(32)$ & 0.71 \\
\hline SAPS2 & $52 \pm 17$ & $47 \pm 13$ & $62 \pm 19$ & $<0.01$ \\
\hline SOFA & $9 \pm 4$ & $7 \pm 3.5$ & $11 \pm 4.5$ & $<0.01$ \\
\hline TIVAP-related infection & $68(45)$ & $55(57)$ & $13(24)$ & $<0.05$ \\
\hline \multicolumn{5}{|l|}{ TIVAP-related infection risk factors, $n$ (\%) } \\
\hline Immunosuppression & $148(98)$ & $97(100)$ & $51(95)$ & 0.99 \\
\hline Hematological malignancies & $72(48)$ & $43(44)$ & $29(54)$ & $<0.05$ \\
\hline Solid organ cancer & $71(47)$ & $52(54)$ & $19(35)$ & 0.12 \\
\hline Metastatic cancer & $44(29)$ & $31(32)$ & $13(24)$ & 0.55 \\
\hline Recent chemotherapy (<6 months) & $131(87)$ & $88(91)$ & $43(80)$ & 0.85 \\
\hline Parenteral nutrition & $18(12)$ & $13(14)$ & $5(9)$ & 0.34 \\
\hline \multicolumn{5}{|l|}{ Initial presentation } \\
\hline Systolic blood pressure, $\mathrm{mmHg}$ & $98 \pm 27$ & $98 \pm 27$ & $101 \pm 28$ & 0.55 \\
\hline Mean blood pressure, mmHg & $69 \pm 20$ & $69 \pm 19$ & $72 \pm 24$ & 0.44 \\
\hline Glasgow Score Scale & $13 \pm 3$ & $14 \pm 3$ & $13 \pm 3$ & 0.20 \\
\hline White blood cells, Giga/l & $7.6 \pm 13.2$ & $7.6 \pm 15.6$ & $7.6 \pm 10.2$ & 0.98 \\
\hline Platelet counts, Giga/l & $116 \pm 113$ & $128 \pm 110$ & $91 \pm 114$ & $<0.05$ \\
\hline Prothrombin time, $\%$ & $64 \pm 17$ & $68 \pm 15$ & $55 \pm 19$ & $<0.01$ \\
\hline Serum creatinine, $\mu \mathrm{mol} / \mathrm{l}$ & $142 \pm 119$ & $125 \pm 106$ & $176 \pm 137$ & 0.01 \\
\hline Bicarbonate, $\mathrm{mmol} / \mathrm{l}$ & $20 \pm 5$ & $21 \pm 6$ & $19 \pm 5$ & $<0.01$ \\
\hline Arterial blood lactate, mmol/l & $3.4 \pm 3.2$ & $2.7 \pm 2.2$ & $5 \pm 4$ & $<0.01$ \\
\hline \multicolumn{5}{|l|}{ Treatments } \\
\hline Time between ICU admission and device withdrawal, days & $1.8 \pm 3.5$ & $1.6 \pm 2.8$ & $2.2 \pm 4.5$ & 0.35 \\
\hline Use of $\mathrm{MV}, n(\%)$ & $74(49)$ & $37(38)$ & $37(69)$ & $<0.01$ \\
\hline MV duration, days & $4 \pm 7$ & $4 \pm 7$ & $5 \pm 5$ & 0.32 \\
\hline Use of vasopressors, $n(\%)$ & $103(68)$ & $62(64)$ & $41(76)$ & $<0.01$ \\
\hline Vasopressors duration, days & $3 \pm 4$ & $2 \pm 3$ & $4 \pm 5$ & $<0.01$ \\
\hline ICU stay, days & $8 \pm 9$ & $9 \pm 10$ & $8 \pm 7$ & 0.53 \\
\hline
\end{tabular}

Categorical variables are expressed as no. (\%) and continuous variables as mean $\pm S D$

ICU intensive care unit, SOFA Sepsis-Related Organ Failure Assessment, SAPS2 Simplified Acute Physiology Score, TIVAP totally implanted venous access port, MV mechanical ventilation

present from 7 to $33 \%$ of the cases $[8-10,17,20]$, in our patient series, only few had local sign of infection (18\%). But it's important to specify that information about local signs of infection is difficult to collect in a retrospective study. They are not systematically looked for and their identification may vary between healthcare providers when clear definitions are not used. Interestingly, it was independently associated with TIVAP-related infection in our study.

\section{TIVAP-related infections: impact on prognosis}

While ICU mortality of sepsis is still high, up to $20 \%$ in latest publications [37-39], it reaches even higher rates in immunocompromised patients, between 40 and 60\% [40]. In the present study, we report a lower mortality: $26 \%$ for ICU mortality and 36\% at 28 days. While expected mortality (predicted by SAPS2 score) was around $40 \%$, patients with TIVAP-related infections had a much lower mortality (9\%). Moreover, ICU mortality was $2 \%$ in patients with exclusive TIVAP-related infection (no other infection focus). TIVAP-related infection was significantly associated with highest ICU and 28-day survival, whereas high SOFA score upon admission and the need for mechanical ventilation were significantly associated with ICU mortality and at 28 days. The low mortality observed in patients with exclusive TIVAP-related infections may be explained by the strong experience in the management of immunocompromised patients of participating ICUs. As a matter of fact, the precociousness of source control was probably a major driver of such a 
favorable outcome. In agreement with other studies, neutropenia was not significantly related to mortality [4144]. Interestingly, patients who had both TIVAP-related infections and another focus had a worst prognosis than patients who had only TIVAP-related infection. In light with this, our study provides an important message for physicians managing immunosuppressed patients with severe presentation (septic shock, need for mechanical ventilation). While admission in the ICU of such patients is sometimes questioned, our findings suggest that in case of TIVAP-related infection, those patients have a short ICU stay and good prognosis. Likewise, our findings suggest that in patients admitted for a suspected TIVAP-related infection who do not recover shortly, this diagnosis should be questioned.

\section{Limits}

While reporting the first cohort of TIVAP-related infections in the ICU, this retrospective study has some limitations. As we obtained data from patient's medical charts, we cannot pretend to be exhaustive in data collection. Important information such as presence of thrombosis or endocarditis associated with TIVAP-related infection would have been interesting, but not all patients had a Doppler ultrasonography at time of admission. Then, it is likely that all patients with TIVAP admitted in participating ICU were not enrolled in the study since the main inclusion criteria was TIVAP removal. As a matter of fact, our inclusion criteria could have biased the true incidence of TIVAP-related infection toward overestimation. Due to the retrospective nature of the study, it was not possible to systematically identify patients who may have been admitted in our ICUs with a TIVAP in place that was not removed. The fact that positive blood cultures were available prior TIVAP removal may have also influenced clinicians' decision. However, ICUs participating in the study had homogeneous practices regarding admission policy of immunocompromised patients and the decision to remove TIVAP in case of life-threatening sepsis. Last, the definition of TIVAP-related infection was extended to patients with positive blood culture without other suspected infection $(n=12)$ and regression of clinical signs of infection after TIVAP removal despite negative culture of TIVAP (catheter tip or the reservoir's port). Since all our patients received antibiotics prior TIVAP removal, a condition that could fairly lead to misclassification, we estimated legitimate to consider these twelve patients as having TIVAP-related infection.

\section{Conclusion}

In almost one out of two cases, TIVAP-related infection was evidenced in immunosuppressed patients admitted in the ICU for sepsis and in whom the device has been removed. With the exception of local signs of infection, no other associated factor could have been identified. TIVAPrelated infection was associated with a good prognosis, as compared to patients with other causes of infection.

\section{Additional file}

Additional file 1: Table 1. Complementary microbiological findings in patients with TIVAP (totally implanted venous-access ports) related infections. Table E2. Complementary microbiological findings in patients without TIVAP (totally implanted venous-access ports) related infections.

Table E3. Variables associated with ICU mortality (univariate analysis). Figure E1. Flow chart of the patients. ICU: intensive care unit; TIVAP: totally implanted venous access ports.

\section{Abbreviations}

TIVAP: totally implanted venous access port; ICU: intensive care unit; SAPS II: Simplified Acute Physiology Score II; SOFA: Sequential Organ Failure Assessment; HIV: human immunodeficiency virus; IDSA: Infectious Diseases Society of America; CFU: colony-forming unit; OR: odd ratio; $\mathrm{Cl}$ : confidence interval.

\section{Authors' contributions}

$M L$ participated in the conception and design of the study, performed the literature search, performed the data analysis and drafted the manuscript. SV helped to collect data. NB helped to collect data. NdeP helped to collect data and helped to revise the manuscript. DR helped to collect data and helped to revise the manuscript. DL, EM, EA and AD helped to revise the manuscript. MD designed the study, helped to draft the manuscript and helped to revise the manuscript. All authors read and approved the final manuscript.

\section{Author details}

1 Service de Pneumologie et Réanimation Médicale (Département "R3S"), Groupe Hospitalier Pitié-Salpêtrière Charles Foix, Assistance Publique-Hôpitaux de Paris, 75013 Paris, France. ${ }^{2}$ Service de Réanimation médicale, Groupe Hospitalier Saint-Louis - Lariboisière - Fernand-Widal, Assistance PubliqueHôpitaux de Paris, Paris, France. ${ }^{3}$ Service de Réanimation médicale, Groupe Hospitalier Est Parisien, Hôpital Saint-Antoine Paris, Assistance PubliqueHôpitaux de Paris, Paris, France. ${ }^{4}$ Service de Réanimation médicale, Groupe Hospitalier Henri Mondor, Assistance Publique-Hôpitaux de Paris, Créteil, France. ${ }^{5}$ Service de Réanimation médico-chirurgicale, Groupe Hospitalier Paris Nord, Hôpital Louis-Mourier, Assistance Publique-Hôpitaux de Paris, Colombes, France. ${ }^{6}$ Service de Microbiologie, Unité Mobile de Microbiologie Clinique, Hôpital Européen Georges Pompidou, Assistance Publique-Hôpitaux de Paris, Paris, France. ${ }^{7}$ Université Paris Descartes, Sorbonne Paris Cité, Paris, France. ${ }^{8}$ INSERM, UMRS1158 Neurophysiologie respiratoire expérimentale et clinique, Sorbonne Universités, UPMC Univ Paris 06, Paris, France.

\section{Acknowledgements}

The authors would like to thank all the microbiologist from each center, for providing the clinical microbiology laboratory data to identify and include the patients.

\section{Competing interests}

Alexandre Demoule has signed research contracts with Medtronic (Dublin, Ireland), Maquet (Solna, Sweden) and Philips (Carlsbad, CA); he has also received personal fees from Medtronic (Dublin, Ireland), Maquet (Solna, Sweden), Fisher\&Paykel (Kingston, Milton Keynes, UK), Resmed, Hamilton and MSD (Courbevoie, France). Martin Dres received personal fees from Pulsion Medical System (Feldkirchen, Germany) and Astra Zeneca (Cambridge, UK). JeanDamien Ricard received travel expenses from Fisher\&Paykel (Kingston, Milton Keynes, UK) to attend scientific meetings. Damien Roux received personal fees from Astellas (Levallois-Perret, France). The other authors declare that they have no competing interests.

Availability of data and materials

The datasets used and/or analyzed during the current study are available from the corresponding author on reasonable request. 


\section{Consent for publication}

Not applicable.

\section{Ethics approval and consent to participate}

The Institutional Review Board of the French Intensive Care Society approved the study (CE SRLF15-52). With respect to the French regulations and with regard to the retrospective nature of the work, no consent to participate was required.

\section{Funding}

MD was supported by the French Intensive Care Society, Paris, France (bourse de mobilité 2015); The 2015 Short Term Fellowship Program of the European Respiratory Society, Lausanne, Switzerland; The 2015 Bernhard Dräger Award for advanced treatment of acute respiratory failure of the European Society of Intensive Care Medicine, Brussels, Belgium; the Assistance Publique-Hôpitaux de Paris, Paris, France; and the Fondation pour la Recherche Médicale, Paris, France (FDM 20150734498).

\section{Publisher's Note}

Springer Nature remains neutral with regard to jurisdictional claims in published maps and institutional affiliations.

Received: 14 January 2018 Accepted: 8 March 2018

Published online: 27 March 2018

\section{References}

1. Niederhuber JE, Ensminger W, Gyves JW, Liepman M, Doan K, Cozzi E. Totally implanted venous and arterial access system to replace external catheters in cancer treatment. Surgery. 1982;92:706-12.

2. Biffi R, Toro A, Pozzi S, Di Carlo I. Totally implantable vascular access devices 30 years after the first procedure. What has changed and what is still unsolved? Support Care Cancer. 2014;22:1705-14.

3. Barbetakis N, Asteriou C, Kleontas A, Tsilikas C. Totally implantable central venous access ports. Analysis of 700 cases. J Surg Oncol. 2011;104:654-6.

4. Groeger JS, Lucas AB, Thaler HT, Friedlander-Klar H, Brown AE, Kiehn $T E$, et al. Infectious morbidity associated with long-term use of venous access devices in patients with cancer. Ann Intern Med. 1993;119:1168-74.

5. Chang L, Tsai J-S, Huang S-J, Shih C-C. Evaluation of infectious complications of the implantable venous access system in a general oncologic population. Am J Infect Control. 2003;31:34-9.

6. Mermel LA, Allon M, Bouza E, Craven DE, Flynn P, O'Grady NP, et al. Clinical practice guidelines for the diagnosis and management of intravascular catheter-related infection: 2009 Update by the Infectious Diseases Society of America. Clin Infect Dis. 2009;49:1-45.

7. Safdar N, Maki DG. Inflammation at the insertion site is not predictive of catheter-related bloodstream infection with short-term, noncuffed central venous catheters. Crit Care Med. 2002;30:2632-5.

8. Whitman ED, Boatman AM. Comparison of diagnostic specimens and methods to evaluate infected venous access ports. Am J Surg. 1995;170:665-9.

9. Douard MC, Arlet G, Longuet P, Troje C, Rouveau M, Ponscarme D, et al. Diagnosis of venous access port-related infections. Clin Infect Dis. 1999;29:1197-202.

10. Hsu J-F, Chang H-L, Tsai M-J, Tsai Y-M, Lee Y-L, Chen P-H, et al. Port type is a possible risk factor for implantable venous access port-related bloodstream infections and no sign of local infection predicts the growth of gram-negative bacilli. World J Surg Oncol. 2015;13:288.

11. Timsit J-F. Updating of the 12th consensus conference of the Société de Réanimation de langue française (SRLF): catheter related infections in the intensive care unit. Ann Fr Anesth Réanim. 2005;24:315-22.

12. Seymour CW, Kahn JM, Martin-Gill C, Callaway CW, Yealy DM, Scales D, et al. Delays from first medical contact to antibiotic administration for sepsis. Crit Care Med. 2017;45:759-65.

13. Bagshaw SM, Lapinsky S, Dial S, Arabi Y, Dodek P, Wood G, et al. Acute kidney injury in septic shock: clinical outcomes and impact of duration of hypotension prior to initiation of antimicrobial therapy. Intensive Care Med. 2009:35:871-81.

14. Kumar A, Roberts D, Wood KE, Light B, Parrillo JE, Sharma S, et al. Duration of hypotension before initiation of effective antimicrobial therapy is the critical determinant of survival in human septic shock. Crit Care Med. 2006;34:1589-96.

15. Lundberg JS, Perl TM, Wiblin T, Costigan MD, Dawson J, Nettleman MD, et al. Septic shock: an analysis of outcomes for patients with onset on hospital wards versus intensive care units. Crit Care Med. 1998;26:1020-4.

16. Lebeaux D, Fernández-Hidalgo N, Chauhan A, Lee S, Ghigo J-M, Almirante $B$, et al. Management of infections related to totally implantable venous-access ports: challenges and perspectives. Lancet Infect Dis. 2014;14:146-59.

17. Lebeaux D, Larroque B, Gellen-Dautremer J, Leflon-Guibout V, Dreyer C, Bialek $\mathrm{S}$, et al. Clinical outcome after a totally implantable venous access port-related infection in cancer patients: a prospective study and review of the literature. Medicine (Baltimore). 2012;91:309-18.

18. Raad I, Hanna H, Maki D. Intravascular catheter-related infections: advances in diagnosis, prevention, and management. Lancet Infect Dis. 2007;7:645-57.

19. Sotir MJ, Lewis C, Bisher EW, Ray SM, Soucie JM, Blumberg HM. Epidemiology of device-associated infections related to a long-term implantable vascular access device. Infect Control Hosp Epidemiol. 1999;20:187-91.

20. Vidal M, Genillon JP, Forestier E, Trouiller S, Pereira B, Mrozek N, et al. Outcome of totally implantable venous-access port-related infections. Méd Mal Infect. 2016:46:32-8.

21. Dellinger RP, Levy MM, Rhodes A, Annane D, Gerlach H, Opal SM, et al. Surviving sepsis campaign: international guidelines for management of severe sepsis and septic shock: 2012. Crit Care Med. 2013;41:580-637.

22. Le Gall JR, Lemeshow S, Saulnier F. A new Simplified Acute Physiology Score (SAPS II) based on a European/North American multicenter study. JAMA. 1993;270:2957-63.

23. Vincent JL, Moreno R, Takala J, Willatts S, De Mendonça A, Bruining $\mathrm{H}$, et al. The SOFA (Sepsis-related Organ Failure Assessment) score to describe organ dysfunction/failure. On behalf of the Working Group on Sepsis-Related Problems of the European Society of Intensive Care Medicine. Intensive Care Med. 1996:22:707-10.

24. Brun-Buisson C, Abrouk F, Legrand P, Huet Y, Larabi S, Rapin M. Diagnosis of central venous catheter-related sepsis. Critical level of quantitative tip cultures. Arch Intern Med. 1987;147:873-7.

25. Cleri DJ, Corrado ML, Seligman SJ. Quantitative culture of intravenous catheters and other intravascular inserts. J Infect Dis. 1980;141:781-6.

26. Safdar N, Fine JP, Maki DG. Meta-analysis: methods for diagnosing intravascular device-related bloodstream infection. Ann Intern Med. 2005; 142:451-66

27. Blot F, Nitenberg G, Chachaty E, Raynard B, Germann N, Antoun S, et al. Diagnosis of catheter-related bacteraemia: a prospective comparison of the time to positivity of hub-blood versus peripheral-blood cultures. Lancet. 1999;354:1071-7.

28. Blot F, Schmidt E, Nitenberg G, Tancrède C, Leclercq B, Laplanche A, et al. Earlier positivity of central-venous-versus peripheral-blood cultures is highly predictive of catheter-related sepsis. J Clin Microbiol. 1998:36:105-9.

29. Pegues D, Axelrod P, McClarren C, Eisenberg BL, Hoffman JP, Ottery FD, et al. Comparison of infections in Hickman and implanted port catheters in adult solid tumor patients. J Surg Oncol. 1992;49:156-62.

30. Rijnders BJ, Van Wijngaerden E, Vandecasteele SJ, Stas M, Peetermans WE. Treatment of long-term intravascular catheter-related bacteraemia with antibiotic lock: randomized, placebo-controlled trial. J Antimicrob Chemother. 2005:55:90-4.

31. Howell PB, Walters PE, Donowitz GR, Farr BM. Risk factors for infection of adult patients with cancer who have tunnelled central venous catheters. Cancer. 1995;75:1367-75.

32. Adler A, Yaniv I, Steinberg R, Solter E, Samra Z, Stein J, et al. Infectious complications of implantable ports and Hickman catheters in paediatric haematology-oncology patients. J Hosp Infect. 2006;62:358-65.

33. Lebeaux D, Zarrouk V, Leflon-Guibout V, Lefort A, Fantin B. Totally implanted access port-related infections: features and management. Rev Méd Interne. 2010;31:819-27. 
34. Penel N, Neu J-C, Clisant S, Hoppe H, Devos P, Yazdanpanah Y. Risk factors for early catheter-related infections in cancer patients. Cancer. 2007;110:1586-92.

35. Santarpia L, Pasanisi F, Alfonsi L, Violante G, Tiseo D, De Simone G, et al. Prevention and treatment of implanted central venous catheter (CVC) related sepsis: a report after six years of home parenteral nutrition (HPN). Clin Nutr Edinb Scotl. 2002;21:207-11.

36. Touré A, Chambrier C, Vanhems P, Lombard-Bohas C, Souquet J-C, Ecochard R. Propensity score analysis confirms the independent effect of parenteral nutrition on the risk of central venous catheter-related bloodstream infection in oncological patients. Clin Nutr Edinb Scotl. 2013;32:1050-4

37. Damiani E, Donati A, Serafini G, Rinaldi L, Adrario E, Pelaia P, et al. Effect of performance improvement programs on compliance with sepsis bundles and mortality: a systematic review and meta-analysis of observational studies. PLoS ONE. 2015;10:e0125827.

38. Kaukonen K-M, Bailey M, Suzuki S, Pilcher D, Bellomo R. Mortality related to severe sepsis and septic shock among critically ill patients in Australia and New Zealand, 2000-2012. JAMA. 2014;311:1308-16.

39. Stevenson EK, Rubenstein AR, Radin GT, Wiener RS, Walkey AJ. Two decades of mortality trends among patients with severe sepsis: a comparative meta-analysis. Crit Care Med. 2014;42:625-31.
40. Pène F, Percheron S, Lemiale V, Viallon V, Claessens Y-E, Marqué S, et al Temporal changes in management and outcome of septic shock in patients with malignancies in the intensive care unit. Crit Care Med. 2008;36:690-6.

41. Azoulay E, Mokart D, Pène F, Lambert J, Kouatchet A, Mayaux J, et al. Outcomes of critically ill patients with hematologic malignancies: prospective multicenter data from France and Belgium-A Groupe de Recherche Respiratoire en Réanimation Onco-Hématologique Study. J Clin Oncol. 2013;31:2810-8.

42. Darmon M, Azoulay E, Alberti C, Fieux F, Moreau D, Le Gall J-R, et al. Impact of neutropenia duration on short-term mortality in neutropenic critically ill cancer patients. Intensive Care Med. 2002;28:1775-80.

43. Mokart D, Darmon M, Resche-Rigon M, Lemiale V, Pène F, Mayaux J, et al. Prognosis of neutropenic patients admitted to the intensive care unit. Intensive Care Med. 2015;41:296-303.

44. Vandijck DM, Benoit DD, Depuydt PO, Offner FC, Blot SI, Van Tilborgh AK, et al. Impact of recent intravenous chemotherapy on outcome in severe sepsis and septic shock patients with hematological malignancies. Intensive Care Med. 2008;34:847-55.

\section{Submit your manuscript to a SpringerOpen ${ }^{\circ}$ journal and benefit from:}

- Convenient online submission

- Rigorous peer review

- Open access: articles freely available online

- High visibility within the field

- Retaining the copyright to your article

Submit your next manuscript at $\boldsymbol{\nabla}$ springeropen.com 